\title{
A Bayesian Analysis of the Spherical Distribution in Presence of Covariates
}

\author{
Jorge Alberto Achcar ${ }^{1}$, Gian Franco Napa ${ }^{1}$ and Roberto Molina De Souza ${ }^{2 *}$ \\ ${ }^{1}$ University of São Paulo and ${ }^{2}$ Federal Technological University of Paraná
}

Abstract: In this paper we introduce a Bayesian analysis of a spherical distribution applied to rock joint orientation data in presence or not of a vector of covariates, where the response variable is given by the angle from the mean and the covariates are the components of the normal upwards vector. Standard simulation MCMC (Markov Chain Monte Carlo) methods have been used to obtain the posterior summaries of interest obtained from WinBugs software. Illustration of the proposed methodology are given using a simulated data set and a real rock spherical data set from a hydroelectrical site.

Key words: Bayesian analysis, MCMC methods, regression models, rock spherical engineering data, spherical distribution.

\section{Introduction}

A distribution of errors over the surface of the unit sphere was introduced by R. A. Fisher in 1952, given by the density,

$$
f(\theta \mid k)=\frac{k}{2 \sinh (k)} \exp (k \cos (\theta)) \sin (\theta),
$$

where $0<\theta<\pi$.

The random variable $\theta$ is the angular displacement from the true position at which $\theta=0$; the parameter $k$ is a measure of precision. The parameter $k$ has the following interpretation:

(i) If $k$ is large the distribution is confined in a small portion of the sphere in the neighborhood of the maximum (small variability);

(ii) If $k=0$ (great variability), the distribution is uniform over all spherical surface.

\footnotetext{
${ }^{*}$ Corresponding author.
} 
Applications of the spherical distribution with density (1) could be considered in different areas, as in geology, where sedimentary rocks could have a great heterogeneity or composition changes in time.

In rock engineering, discontinuities, also known as joints, are idealized as planes that interrupt the continuity of a rock mass. The representation of an individual joint is made through the vector perpendicular to itself. A rock mass can be intersected by many sub parallel planes that are clustered in groups denominated as families. Moreover, a rock mass usually presents more than one family of discontinuities. These families are typically represented following Fisher distributions (Merrien-Soukatchoff et al., 2012).

However, in some cases Fisher distribution cannot adjust field data (Lanney, 1978). This fact can be originated from the presence of some covariates that not were appropriately identified and treated. The presence of covariates such as spatial position transforms the random variable vector problem into a stochastic process and the treatment needs to be different.

In rock mechanics, we can find some problems where we need to estimate the reliability of some performance functions at spatial location that we have not reached yet, e.g., tunneling. In this case Bayesian prediction constitutes a strong tool to perform this task. There exists some approaches for performing Bayesian prediction of the rock mass conditions ahead of the face of the excavation but these are planned for equivalent continuous rock masses (Einstein, 2004). At present, we do not have a Bayesian prediction tool for jointed systems.

Consequently, the scope of this paper is to offer a statistical tool to evaluate the presence of covariates in a joint cluster. The application of Bayesian prediction will be presented in another engineering paper.

In Figure 1, we have plots the spherical density (1) considering different values of $k$. The mean of the spherical distribution with density (1) is given (see Appendix 1) by,

$$
E(\theta \mid k)=\frac{\pi}{\exp (k)-\exp (-k)}\left[I_{0}(k)-\exp (-k)\right],
$$

where $I_{0}(k)=\sum_{m=0}^{\infty} 1 / m ! \Gamma(m+1)(k / 2)^{2 m}$ is the modified Bessel function of first kind (see Abramowitz and Stegun, 1965). by

An approximation for $(2)$ considering $I_{0}(k) \cong 1+(k / 2)^{2}+1 / 4(k / 2)^{4}$ is given

$$
E(\theta \mid k) \cong \frac{\pi k}{\exp (k)-\exp (-k)}\left[\frac{k^{3}}{64}+\frac{k}{4}+\frac{1}{k}-\frac{\exp (-k)}{k}\right] .
$$

In Table 1, we have some approximations for $E(\theta \mid k)$ using (3) for different values of $k$. 


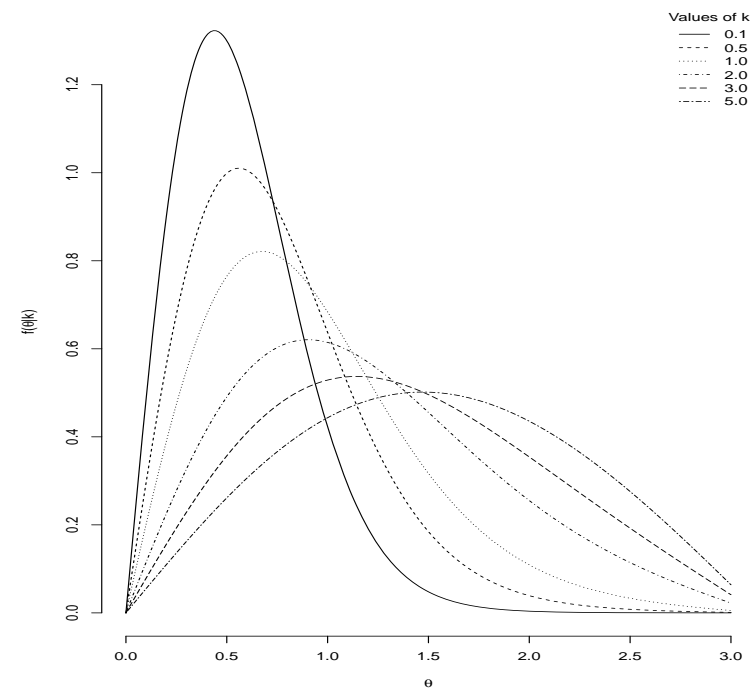

Figure 1: Spherical densities for different values of $k$

Table 1: Approximated values for $E(\theta \mid k)$

\begin{tabular}{rcccccc}
\hline$k$ & 5 & 3 & 2 & 1 & 0.5 & 0.1 \\
\hline$E(\theta \mid k)$ & 0.36009 & 0.70024 & 0.91586 & 1.19994 & 1.37742 & 1.53155 \\
\hline
\end{tabular}

The paper is organized as follows: in Section 2, we introduce the likelihood function; in Section 3, we present a Bayesian analysis for the model; in Section 4, we assume the presence of covariates; in Section 5 we introduce two examples considering a simulated data set and a real rock engineering data set in presence of covariates; finally, in Section 6, we present some concluding remarks.

\section{The Likelihood Function for $k$}

Let us assume that $\boldsymbol{\theta}=\left(\theta_{1}, \cdots, \theta_{n}\right)$ is a vector denoting a random sample of size $n$ of the spherical distribution with density (1).

The likelihood function for the parameter $k$ is given by, $L(k)=\prod_{i=1}^{n} f\left(\theta_{i} \mid k\right)$ where $f\left(\theta_{i} \mid k\right)$ is given by (1). That is,

$$
L(k)=\left[\frac{k}{2 \sinh (k)}\right] \exp (k x)\left[\prod_{i=1}^{n} \sin \left(\theta_{i}\right)\right],
$$

where $x=\sum_{i=1}^{n} \cos \left(\theta_{i}\right)$.

The logarithm of the likelihood function (4) is given by, 


$$
l(k)=\log [L(k)]=n \log (k)-n \log [2 \sinh (k)]+k x-\sum_{i=1}^{n} \log \left[\sin \left(\theta_{i}\right)\right] .
$$

The first derivate of the logarithm of the likelihood function with respect to $k$ is given by, $d l / d k=n / k-n \cosh (k) / \sinh (k)+x=0$. Since $\operatorname{coth}(k)=$ $\cosh (k) / \sinh (k)$ and $\operatorname{coth}(k)=[\exp (k)+\exp (-k)] /[\exp (k)-\exp (-k)]$, we get the maximum likelihood estimator of $k$ from the equation,

$$
h(k)=\frac{n}{k}-n \frac{\exp (k)+\exp (-k)}{\exp (k)-\exp (-k)}+x=0,
$$

where $x=\sum_{i=1}^{n} \cos \left(\theta_{i}\right)$.

The maximum likelihood estimator for $k$ is obtained using a numerical iterative method (for example, the Newton-Raphson algorithm).

Using the invariance property of maximum likelihood estimators (see Casella and Berger, 2002, Chapter 7), the maximum likelihood estimator (MLE) for the mean of the spherical distribution with density (1) is given by,

$$
\widehat{E(\theta \mid k)}=\frac{\pi \hat{k}}{\hat{k}[\exp (\hat{k})-\exp (-\hat{k})]}\left[I_{0}(\hat{k})-\exp (-\hat{k})\right],
$$

(see (2)), where $I_{0}(k)$ is the modified Bessel function of first kind.

\section{A Bayesian Analysis}

Let us assume a non-informative prior distribution (see Box and Tiao, 1992; Bernardo and Smith, 2007, Chapter 5); for $k$ given by,

$$
p(k) \propto \frac{1}{k},
$$

where $k>0$.

Combining the prior (8) with likelihood function for $k(4)$, we get from the Bayes formula, the posterior distribution for $k$ given by,

$$
p(k \mid \boldsymbol{\theta})=c \frac{k^{n-1} \exp (k x)}{[2 \sinh (k)]^{n}},
$$

where $k>0, x=\sum_{i=1}^{n} \cos \left(\theta_{i}\right), 2 \sinh (k)=\exp (k)-\exp (-k)$ and $c$ is the normalizing constant given by $c^{-1}=\int_{0}^{\infty} k^{(n-1)} \exp (k x) /[\exp (k)-\exp (-k)]^{n} d k$.

A Bayesian estimator with respect to the squared error loss function for $k$ is given by the posterior mean,

$$
E(k \mid \boldsymbol{\theta})=c \int_{0}^{\infty} \frac{k^{(n)} \exp (k x)}{[\exp (k)-\exp (-k)]^{n}} d k .
$$


The posterior mean for $k$ (10) given a data set $\boldsymbol{\theta}=\left(\theta_{1}, \cdots, \theta_{n}\right)$ could be calculated using standard Monte Carlo methods or using approximation methods for integrals.

Using Laplace's method (see Tierney and Kadane, 1986; Tierney et al., 1989), we can rewrite (10) as,

$$
E(k \mid \boldsymbol{\theta})=\frac{\int_{0}^{\infty} \exp \left(-n h^{*}(k)\right) d k}{\int_{0}^{\infty} \exp (-n h(k)) d k},
$$

where $-n h^{*}(k)=n \log (k)+k x-n \log [\exp (k)-\exp (-k)]$ and $-n h(k)=(n-$ 1) $\log (k)+k x-n \log [\exp (k)-\exp (-k)]$.

The standard Laplace's approximation for integrals is given by,

$$
\int_{-\infty}^{\infty} \exp (-n h(k)) d k \cong \sqrt{2 \pi} \exp (-n h(\hat{k}))\left(-n h^{\prime \prime}(\hat{k})\right)^{\frac{1}{2}},
$$

where $\hat{k}$ is the maximum of $-n h(k)$, that is, $-n h^{\prime}(\hat{k})=0$, and $h^{\prime \prime}(\hat{k})$ is the second derivate of $h(k)$.

Using the Laplace's approximation (12) in the numerator and denominator of (11), we get the approximation,

$$
\begin{aligned}
\widehat{E(\theta \mid k)} \cong & \frac{\left(k^{*}\right)^{n} \exp \left(k^{*} x\right)[\exp (\hat{k})-\exp (-\hat{k})]^{n}}{\hat{k}^{(n-1)} \exp (\hat{k} x)\left[\exp \left(\hat{k}^{*}\right)-\exp \left(-\hat{k}^{*}\right)\right]^{n}} \\
& \times \frac{\left[\frac{n}{\left(\hat{k}^{*}\right)^{2}}-\frac{4 n}{\left[\exp \left(\hat{k}^{*}\right)-\exp \left(-\hat{k}^{*}\right)\right]^{2}}\right]^{\frac{1}{2}}}{\left[\frac{n-1}{(\hat{k})^{2}}-\frac{4 n}{[\exp (\hat{k})-\exp (-\hat{k})]^{2}}\right]^{\frac{1}{2}}}
\end{aligned}
$$

where $\hat{k}^{*}$ is given by $n / \hat{k}^{*}+x-n\left[\exp \left(\hat{k}^{*}\right)+\exp \left(-\hat{k}^{*}\right)\right] /\left[\exp \left(\hat{k}^{*}\right)-\exp \left(-\hat{k}^{*}\right)\right]=0$ and $\hat{k}$ is given by $n-1 / \hat{k}+x-n[\exp (\hat{k})+\exp (-\hat{k})] /[\exp (\hat{k})-\exp (-\hat{k})]=0$ and $x=\sum_{i=1}^{n} \cos \left(\theta_{i}\right)$.

\section{Presence of Covariates}

In presence of one or more covariates, we could assume the following regression model:

$$
k_{i}=\exp \left(\boldsymbol{X}_{i}^{\prime} \boldsymbol{\beta}\right)
$$

where $\boldsymbol{X}_{i}^{\prime}=\left(1, X_{1_{i}}, \cdots, X_{p_{i}}\right)$ is a vector of covariates and $\boldsymbol{\beta}=\left(\beta_{0}, \beta_{1}, \cdots, \beta_{p}\right)$ is a vector of regression parameters, that is, $\boldsymbol{X}_{i}^{\prime} \boldsymbol{\beta}=\beta_{0}+\beta_{1} X_{1_{i}}+\cdots+\beta_{p} X_{p_{i}}$, $i=1, \cdots, n$. 
For a Bayesian analysis, let us assume the following prior distribution for the regression parameters:

$$
\begin{aligned}
& \beta_{0} \sim \mathcal{N}\left(a_{0} ; b_{0}^{2}\right), \\
& \beta_{j} \sim \mathcal{N}\left(0 ; b_{j}^{2}\right),
\end{aligned}
$$

where $j=1, \cdots, p ; \mathcal{N}\left(\mu, \sigma^{2}\right)$ denotes a normal distribution with mean equals to $\mu$ and variance equals to $\sigma^{2} ; a_{0}, b_{0}$ and $b_{j}$ are known hyperparameters.

The joint posterior distribution for $\boldsymbol{\beta}$ assuming the prior distributions (15) and prior independence is given by,

$$
\begin{aligned}
p(\boldsymbol{\beta} \mid \boldsymbol{\theta}) \propto & \exp \left(-\frac{1}{2 b_{0}^{2}}\left(\beta_{0}-a_{0}\right)^{2}\right) \prod_{j=1}^{p} \exp \left(-\frac{\beta_{j}^{2}}{2 b_{j}^{2}}\right) \\
& \times \exp \left(\sum_{i=1}^{n} k_{i} \cos \left(\theta_{i}\right)\right) \prod_{i=1}^{n}\left\{\frac{k_{i}}{\exp \left(k_{i}\right)-\exp \left(-k_{i}\right)}\right\},
\end{aligned}
$$

where $k_{i}$ is given by (14).

Posterior summaries of interest for the joint posterior distribution (16) could be obtained using standard MCMC (Markov Chain Monte Carlo) methods (see Gelfand and Smith, 1990; Chib and Greenberg, 1995).

\section{Examples}

\subsection{A Simulated Data Set}

The accumulated distribution function of the spherical distribution with density (1) is given by

$$
F(\theta \mid k)=\left[\frac{k}{2 \sinh (k)}\right] \int_{0}^{\theta} \exp (k \cos (u)) \sin (u) d u,
$$

where $0<\theta<\pi$. That is, $F(\theta \mid k)=(\exp (k)-\exp (k \cos (\theta))) /(\exp (k)-$ $\exp (-k))$.

Since $F(\theta \mid k) \sim \mathcal{U}(0,1)$, where $\mathcal{U}(0,1)$ denotes an uniform distribution in the interval $(0,1)$, we simulate a value of the uniform distribution $\mathcal{U}(0,1)$, that is, $X_{1_{i}}$ in this way,

$$
X_{1}=\frac{\exp (k)-\exp \left(k \cos \left(\theta_{1}\right)\right)}{\exp (k)-\exp (-k)}
$$

where $\theta_{1}=\arccos \left\{1 / k \log \left[\exp (k)-X_{1} \exp (k)+X_{1} \exp (-k)\right]\right\}$.

In Table 2, we have a simulated data set of $n=50$ observations with $k=0.8$ (use of R Software (R Development Core Team, 2012)). 
Table 2: A simulated data set of size $n=50$ assuming $k=0.8$

\begin{tabular}{lllll}
\hline 1.19865 & 1.66331 & 0.50804 & 0.51265 & 1.74771 \\
0.48670 & 2.11942 & 1.52889 & 1.62911 & 1.59583 \\
0.54661 & 1.35978 & 1.97644 & 1.14638 & 0.77208 \\
1.30261 & 0.68733 & 0.74019 & 0.49657 & 2.28964 \\
0.54474 & 1.77158 & 0.45094 & 1.22844 & 0.73070 \\
0.80016 & 1.99580 & 1.30424 & 0.30916 & 1.69721 \\
0.47143 & 1.91275 & 1.61882 & 0.86586 & 1.59613 \\
1.16967 & 1.57620 & 0.90435 & 2.08444 & 1.34750 \\
2.29437 & 2.22655 & 1.53593 & 0.29195 & 0.51069 \\
2.32105 & 1.30503 & 2.26638 & 2.68381 & 1.03450 \\
\hline
\end{tabular}

From (6), we get the MLE for $k$ given by $\hat{k}=0.6785$. From (3), the MLE for the mean of the spherical distribution with density (1) is given by $\widehat{E(\theta \mid k)} \cong$ 0.346894 .

Considering a Bayesian analysis, with prior (8) for $k$, the Laplace's approximation for the posterior mean of $k$ is given by (13), where $\hat{k}^{*}=0.6785$ and $\hat{k}=0.5638$. From $(13)$, we get $\widehat{E(k \mid \theta)} \cong 0.6885$. This value is close of the MLE because we use non informative priors.

We also get Monte Carlos estimates for $k$ and the mean $\mu=E(\theta \mid k)$ (see (3)) using MCMC methods. In Table 3, we have the posterior summaries of interest considering two different priors for $k: k \sim \mathcal{U}(0,1000)$ (prior 1) and $k \sim$ $\operatorname{Gamma}(0.8,1)$ (prior 2) where $\operatorname{Gamma}(a, b)$ denotes a gamma prior with mean $a / b$ and variance $a / b^{2}$. The Monte Carlo estimates were obtained using the WinBUGS software (Lunn et al., 2000) considering 1000 simulated samples for the posterior distribution of $k$ after "burn-in-sample" period of 3000 simulated samples discarded to eliminate the effect of the initial values in the iterative simulation procedure.

Table 3: Posterior summaries

\begin{tabular}{ccccc}
\hline & parameter & mean & S. D. & $95 \%$ credible interval \\
\hline prior 1 & $k$ & 0.6918 & 0.2611 & $(0.1772,1.209)$ \\
& $\mu$ & 1.31 & 0.09344 & $(1.134,1.504)$ \\
\hline prior 2 & $k$ & 0.5928 & 0.2609 & $(0.1035,1.079)$ \\
& $\mu$ & 1.345 & 0.09531 & $(1.175,1.531)$ \\
\hline
\end{tabular}

From the results of Table 3, we observe similar results assuming a more noninformative prior 1 and the informative prior 2 . 


\subsection{A Real Data Set}

We used one data set from the joint families identified after clustering analyses of the geological orientation data (Priest, 1985, 1993). It was found that the mean plane orientation was $138.43^{\circ}$ for dip direction and dipping $66.56^{\circ}$. This leads us to a mean upwards vector orientation of $318.43^{\circ}$ and dip $23.44^{\circ}$ or $(-0.6088,0.6864,0.3978)$ in Cartesian coordinates. Appendix 2 presents orientation data relative to this mean vector. In Figure 2, we have the plots of the angles versus each component of the normal upwards vector.
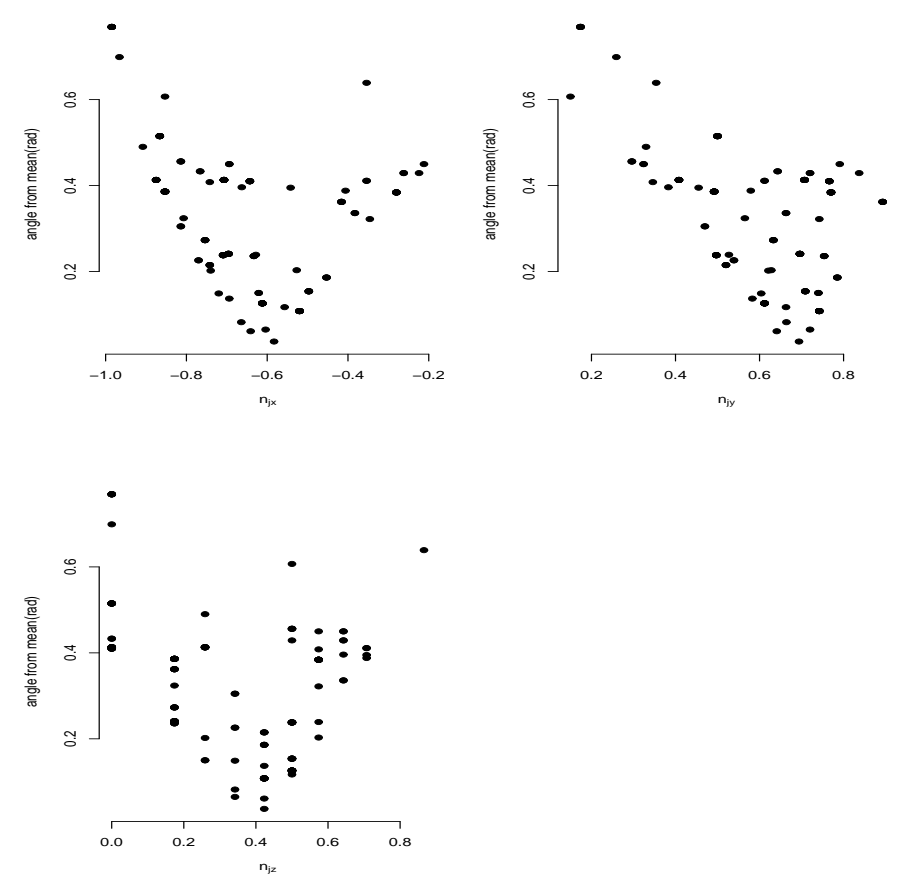

Figure 2: Angles versus each component of the normal upwards vector

From the graphs in Figure 2, we observe that the components of the normal upwards vector apparently affect the angles from the mean. To analyse the data set of Table 4, let us assume the spherical distribution with density (1) and the regression model (14), that is,

$$
k_{i}=\exp \left(\beta_{0}+\beta_{1} n . x_{i}+\beta_{2} n . y_{i}+\beta_{3} n . z_{i}\right),
$$

where $i=1, \cdots, 153$.

We develop a Bayesian analysis of the model assuming the normal prior distributions (15) for the regression parameters $\beta_{0}, \beta_{1}, \beta_{2}$ and $\beta_{3}$ with the following hyperparameter values: $a_{0}=0, b_{0}^{2}=10$ and $b_{j}^{2}=10, j=1,2,3$. Observe that 
Table 4: Posterior summaries (presence of covariates)

\begin{tabular}{ccccc}
\hline parameter & mean & standard deviation & \multicolumn{2}{c}{$95 \%$ credible interval } \\
\hline$\beta_{0}$ & -7.026 & 1.453 & -9.922 & -4.34 \\
$\beta_{1}$ & -6.426 & 1.028 & -8.496 & -4.45 \\
$\beta_{2}$ & 7.363 & 1.064 & 5.414 & 9.64 \\
$\beta_{3}$ & 4.61 & 0.6857 & 3.28 & 6.056 \\
\hline
\end{tabular}

we are using approximately non-informative priors for each parameter. In Table 4, we have the posterior summaries of interest obtained from a final simulated Gibbs sample of size 1,000 using the WinBUGS software with a "burn-in-sample" of size 3,000 and taking every $30^{\text {th }}$ simulated sample to have approximately uncorrelated samples used to get the Monte Carlo estimates for the parameters. Convergence of the algorithm was obtained observing traceplots of the simulated Gibbs samples. The code of the WinBUGS software used in this example is given in Appendix 3 at the end of the paper.

From the Monte Carlo estimates for the regression parameters based on the 1,000 simulated Gibbs samples, we observe that the components of the normal upwards vector affect the angles from the mean, since zero is not included in each $95 \%$ credible interval for the regression parameters $\beta_{0}, \beta_{1}, \beta_{2}$ and $\beta_{3}$. That is, the components of the normal upwards vector affect the precision parameter of the spherical distribution.

\section{Concluding Remarks}

The use of Bayesian methods could be a good alternative to analyze spherical data, especially using MCMC methods. We also observe that the use of existing available software as the WinBUGS software gives great simplification in the simulation of samples of the joint posterior distribution of interest, especially in presence of a covariate vector. When we do not have the presence of covariates, the use of Laplace's method (see (13)) is a good alternative to get accurate estimates for the mean of the spherical distribution with density (1). These results could be of great interest for rock engineerings working with spherical data as observed in the applications section with simulated and real data set.

\section{Appendix 1: The Mean of the Spherical Distribution with Density (1)}

Since the hyperbolic trigonometric function $\sinh (k)=[\exp (k)-\exp (-k)] / 2$, the mean of the spherical distribution with density (1) is given by

$$
E(\theta \mid k)=\frac{k}{\exp (k)-\exp (-k)} \int_{0}^{\pi} \theta \exp (k \cos (\theta)) \sin (\theta) d \theta .
$$


Considering the transformation $y=\cos (\theta)$, that is, $d y=-\sin (\theta) d \theta$, we have $\theta=\arccos (y),-1<y<1$, that is,

$$
E(\theta \mid k)=\frac{k}{\exp (k)-\exp (-k)} \int_{-1}^{1} \arccos (y) \exp (k y) d y .
$$

To solve the integral in (A.2) we use integration by parts,

$$
\int u d v=u v-\int v d u
$$

where $u=\arccos (y)$ and $\exp (k y) d y=d v$, that is, $v=\exp (k y) / k$ and $d u=$ $d y / \sqrt{1-y^{2}}$. In this way,

$$
\int_{-1}^{1} \arccos (y) \exp (k y) d y=\frac{\arccos (y) \exp (k y)}{k} \int_{-1}^{1}+\frac{1}{k} \int_{-1}^{1} \frac{\exp (k y) d y}{\sqrt{1-y^{2}}} .
$$

That is,

$$
\int_{-1}^{1} \arccos (y) \exp (k y) d y=-\frac{\pi \exp (-k)}{k}+\frac{1}{k} \int_{-1}^{1} \frac{\exp (k y) d y}{\sqrt{1-y^{2}}} .
$$

To solve the integral in (A.5), we assume transformation $z=\arccos (y)(y=$ $\cos (z))$. where $1-y^{2}=\sin ^{2}(z)$ and $d y=-\sin (z) d z ;$ that is,

$$
\int_{-1}^{1} \frac{\exp (k y) d y}{\sqrt{1-y^{2}}} d z=\int_{0}^{\pi} \frac{\exp (k \cos (z))}{\sin (z)} \sin (z) d z=\int_{0}^{\pi} \exp (k \cos (z))=\pi I_{0}(k),
$$

where $I_{0}(k)$ is the modified Bessel function or first kind, given by,

$$
I_{0}(k)=\sum_{m=0}^{\infty} \frac{1}{m ! \Gamma(m+1)}\left(\frac{k}{2}\right)^{2 m} .
$$

From (A.2), (A.5) and (A.7), we get,

$$
E(\theta \mid k)=\frac{\pi k}{\exp (k)-\exp (-k)}\left[I_{0}(k)-\exp (-k)\right] .
$$

\section{Appendix 2: Real Data}


Table 5: A orientation data set of the discontinuities of a hydrological dam

\begin{tabular}{|c|c|c|c|c|c|c|c|}
\hline \multicolumn{3}{|c|}{ normal upwards vector } & \multirow{2}{*}{$\begin{array}{l}\text { from mean } \\
\text { (angle) }\end{array}$} & \multicolumn{3}{|c|}{ normal upwards vector } & \multirow{2}{*}{$\begin{array}{c}\text { from mean } \\
\text { (angle) }\end{array}$} \\
\hline$n_{j x}$ & $n_{j y}$ & $n_{j z}$ & & $n_{j x}$ & $n_{j y}$ & $n_{j z}$ & \\
\hline-0.416 & 0.893 & 0.174 & 0.362 & -0.866 & 0.5 & 0 & 0.515 \\
\hline-0.416 & 0.893 & 0.174 & 0.362 & -0.866 & 0.5 & 0 & 0.515 \\
\hline-0.416 & 0.893 & 0.174 & 0.362 & -0.866 & 0.5 & 0 & 0.515 \\
\hline-0.416 & 0.893 & 0.174 & 0.362 & -0.866 & 0.5 & 0 & 0.515 \\
\hline-0.866 & 0.5 & 0 & 0.515 & -0.557 & 0.663 & 0.5 & 0.117 \\
\hline-0.354 & 0.354 & 0.866 & 0.639 & -0.814 & 0.47 & 0.342 & 0.305 \\
\hline-0.754 & 0.633 & 0.174 & 0.273 & -0.814 & 0.47 & 0.342 & 0.305 \\
\hline-0.754 & 0.633 & 0.174 & 0.273 & -0.612 & 0.612 & 0.5 & 0.126 \\
\hline-0.754 & 0.633 & 0.174 & 0.273 & -0.853 & 0.492 & 0.174 & 0.386 \\
\hline-0.754 & 0.633 & 0.174 & 0.273 & -0.853 & 0.492 & 0.174 & 0.386 \\
\hline-0.664 & 0.664 & 0.342 & 0.082 & -0.853 & 0.492 & 0.174 & 0.386 \\
\hline-0.612 & 0.612 & 0.5 & 0.126 & -0.853 & 0.492 & 0.174 & 0.386 \\
\hline-0.612 & 0.612 & 0.5 & 0.126 & -0.52 & 0.742 & 0.423 & 0.108 \\
\hline-0.612 & 0.612 & 0.5 & 0.126 & -0.52 & 0.742 & 0.423 & 0.108 \\
\hline-0.612 & 0.612 & 0.5 & 0.126 & -0.52 & 0.742 & 0.423 & 0.108 \\
\hline-0.612 & 0.612 & 0.5 & 0.126 & -0.52 & 0.742 & 0.423 & 0.108 \\
\hline-0.612 & 0.612 & 0.5 & 0.126 & -0.52 & 0.742 & 0.423 & 0.108 \\
\hline-0.612 & 0.612 & 0.5 & 0.126 & -0.52 & 0.742 & 0.423 & 0.108 \\
\hline-0.612 & 0.612 & 0.5 & 0.126 & -0.52 & 0.742 & 0.423 & 0.108 \\
\hline-0.612 & 0.612 & 0.5 & 0.126 & -0.52 & 0.742 & 0.423 & 0.108 \\
\hline-0.694 & 0.324 & 0.643 & 0.45 & -0.52 & 0.742 & 0.423 & 0.108 \\
\hline-0.694 & 0.324 & 0.643 & 0.45 & -0.52 & 0.742 & 0.423 & 0.108 \\
\hline-0.707 & 0.707 & 0 & 0.413 & -0.853 & 0.492 & 0.174 & 0.386 \\
\hline-0.707 & 0.707 & 0 & 0.413 & -0.853 & 0.492 & 0.174 & 0.386 \\
\hline-0.707 & 0.707 & 0 & 0.413 & -0.853 & 0.492 & 0.174 & 0.386 \\
\hline-0.707 & 0.707 & 0 & 0.413 & -0.853 & 0.492 & 0.174 & 0.386 \\
\hline-0.707 & 0.707 & 0 & 0.413 & -0.853 & 0.492 & 0.174 & 0.386 \\
\hline-0.707 & 0.707 & 0 & 0.413 & -0.853 & 0.492 & 0.174 & 0.386 \\
\hline-0.814 & 0.296 & 0.5 & 0.456 & -0.643 & 0.766 & 0 & 0.41 \\
\hline-0.742 & 0.346 & 0.574 & 0.408 & -0.643 & 0.766 & 0 & 0.41 \\
\hline-0.814 & 0.296 & 0.5 & 0.456 & -0.643 & 0.766 & 0 & 0.41 \\
\hline-0.814 & 0.296 & 0.5 & 0.456 & -0.643 & 0.766 & 0 & 0.41 \\
\hline-0.663 & 0.383 & 0.643 & 0.396 & -0.346 & 0.742 & 0.574 & 0.322 \\
\hline-0.633 & 0.754 & 0.174 & 0.236 & -0.966 & 0.259 & 0 & 0.699 \\
\hline-0.604 & 0.72 & 0.342 & 0.065 & -0.212 & 0.791 & 0.574 & 0.45 \\
\hline-0.74 & 0.621 & 0.259 & 0.202 & -0.908 & 0.33 & 0.259 & 0.49 \\
\hline-0.612 & 0.612 & 0.5 & 0.126 & -0.542 & 0.455 & 0.707 & 0.395 \\
\hline-0.866 & 0.5 & 0 & 0.515 & -0.707 & 0.707 & 0 & 0.413 \\
\hline-0.866 & 0.5 & 0 & 0.515 & -0.707 & 0.707 & 0 & 0.413 \\
\hline
\end{tabular}


830 Jorge Alberto Achcar, Gian Franco Napa and Roberto Molina De Souza

Table 6: A orientation data set of the discontinuities of a hydrological dam

\begin{tabular}{|c|c|c|c|c|c|c|c|}
\hline \multicolumn{3}{|c|}{ normal upwards vector } & \multirow{2}{*}{$\begin{array}{l}\text { from mean } \\
\text { (angle) }\end{array}$} & \multicolumn{3}{|c|}{ normal upwards vector } & \multirow{2}{*}{$\begin{array}{c}\text { from mean } \\
\text { (angle) }\end{array}$} \\
\hline$n_{j x}$ & $n_{j y}$ & $n_{j z}$ & & $n_{j x}$ & $n_{j y}$ & $n_{j z}$ & \\
\hline-0.707 & 0.707 & 0 & 0.413 & -0.28 & 0.77 & 0.574 & 0.384 \\
\hline-0.766 & 0.643 & 0 & 0.433 & -0.28 & 0.77 & 0.574 & 0.384 \\
\hline-0.766 & 0.643 & 0 & 0.433 & -0.28 & 0.77 & 0.574 & 0.384 \\
\hline-0.985 & 0.174 & 0 & 0.769 & -0.28 & 0.77 & 0.574 & 0.384 \\
\hline-0.985 & 0.174 & 0 & 0.769 & -0.383 & 0.663 & 0.643 & 0.336 \\
\hline-0.985 & 0.174 & 0 & 0.769 & -0.383 & 0.663 & 0.643 & 0.336 \\
\hline-0.406 & 0.579 & 0.707 & 0.388 & -0.224 & 0.837 & 0.5 & 0.429 \\
\hline-0.527 & 0.628 & 0.574 & 0.203 & -0.633 & 0.754 & 0.174 & 0.236 \\
\hline-0.709 & 0.497 & 0.5 & 0.238 & -0.633 & 0.754 & 0.174 & 0.236 \\
\hline-0.709 & 0.497 & 0.5 & 0.238 & -0.453 & 0.785 & 0.423 & 0.186 \\
\hline-0.742 & 0.52 & 0.423 & 0.215 & -0.453 & 0.785 & 0.423 & 0.186 \\
\hline-0.742 & 0.52 & 0.423 & 0.215 & -0.453 & 0.785 & 0.423 & 0.186 \\
\hline-0.742 & 0.52 & 0.423 & 0.215 & -0.621 & 0.74 & 0.259 & 0.15 \\
\hline-0.875 & 0.408 & 0.259 & 0.413 & -0.621 & 0.74 & 0.259 & 0.15 \\
\hline-0.875 & 0.408 & 0.259 & 0.413 & -0.354 & 0.612 & 0.707 & 0.411 \\
\hline-0.875 & 0.408 & 0.259 & 0.413 & -0.354 & 0.612 & 0.707 & 0.411 \\
\hline-0.875 & 0.408 & 0.259 & 0.413 & -0.643 & 0.766 & 0 & 0.41 \\
\hline-0.875 & 0.408 & 0.259 & 0.413 & -0.853 & 0.15 & 0.5 & 0.607 \\
\hline-0.875 & 0.408 & 0.259 & 0.413 & -0.641 & 0.641 & 0.423 & 0.061 \\
\hline-0.875 & 0.408 & 0.259 & 0.413 & -0.497 & 0.709 & 0.5 & 0.154 \\
\hline-0.77 & 0.539 & 0.342 & 0.226 & -0.497 & 0.709 & 0.5 & 0.154 \\
\hline-0.77 & 0.539 & 0.342 & 0.226 & -0.72 & 0.604 & 0.342 & 0.149 \\
\hline-0.985 & 0.174 & 0 & 0.769 & -0.807 & 0.565 & 0.174 & 0.324 \\
\hline-0.985 & 0.174 & 0 & 0.769 & -0.583 & 0.694 & 0.423 & 0.037 \\
\hline-0.866 & 0.5 & 0 & 0.515 & -0.696 & 0.696 & 0.174 & 0.241 \\
\hline-0.416 & 0.893 & 0.174 & 0.362 & -0.696 & 0.696 & 0.174 & 0.241 \\
\hline-0.694 & 0.583 & 0.423 & 0.137 & -0.696 & 0.696 & 0.174 & 0.241 \\
\hline-0.985 & 0.174 & 0 & 0.769 & -0.696 & 0.696 & 0.174 & 0.241 \\
\hline-0.709 & 0.497 & 0.5 & 0.238 & -0.696 & 0.696 & 0.174 & 0.241 \\
\hline-0.643 & 0.766 & 0 & 0.41 & -0.696 & 0.696 & 0.174 & 0.241 \\
\hline-0.497 & 0.709 & 0.5 & 0.154 & -0.696 & 0.696 & 0.174 & 0.241 \\
\hline-0.262 & 0.72 & 0.643 & 0.429 & -0.709 & 0.497 & 0.5 & 0.238 \\
\hline-0.262 & 0.72 & 0.643 & 0.429 & -0.709 & 0.497 & 0.5 & 0.238 \\
\hline-0.628 & 0.527 & 0.574 & 0.239 & -0.709 & 0.497 & 0.5 & 0.238 \\
\hline-0.28 & 0.77 & 0.574 & 0.384 & -0.709 & 0.497 & 0.5 & 0.238 \\
\hline-0.28 & 0.77 & 0.574 & 0.384 & -0.497 & 0.709 & 0.5 & 0.154 \\
\hline-0.28 & 0.77 & 0.574 & 0.384 & -0.497 & 0.709 & 0.5 & 0.154 \\
\hline-0.28 & 0.77 & 0.574 & 0.384 & - & - & - & - \\
\hline
\end{tabular}




\section{Appendix 3: WinBUGS Code}

Listing 1: WinBUGS code

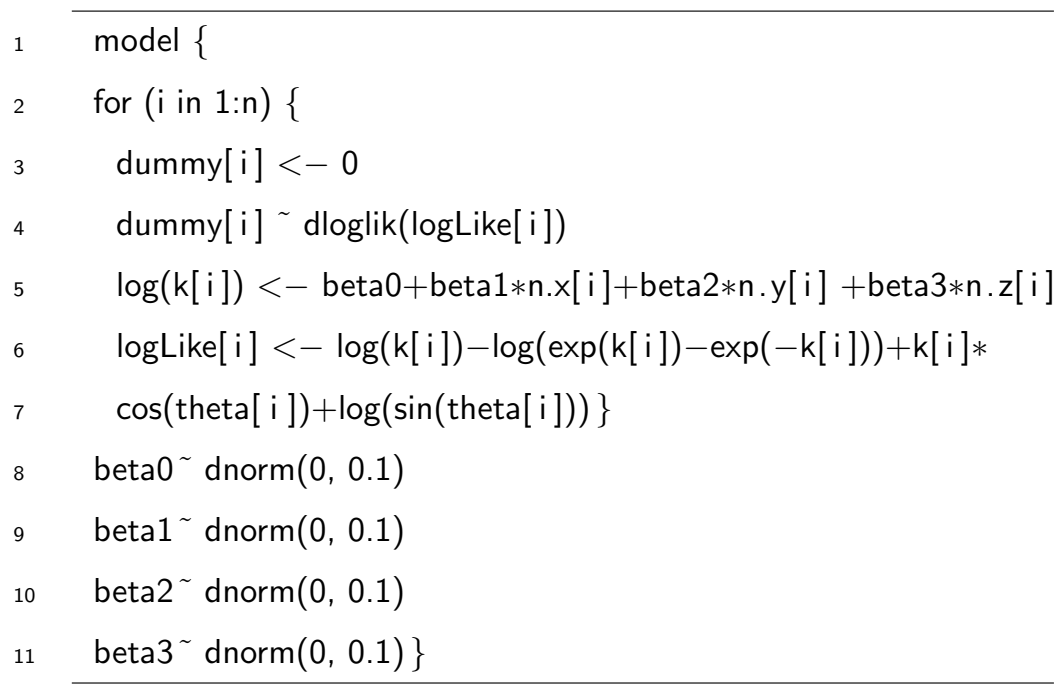

\section{References}

Abramowitz, M. and Stegun, I. A. (1965). Handbook of Mathematical Functions: With Formulas, Graphs, and Mathematical Tables. Applied Mathematics Series. Dover Publications, New York.

Bernardo, J. M. and Smith, A. F. M. (2007). Bayesian Theory, 2nd edition. Wiley Series in Probability and Statistics. Wiley, New York.

Box, G. E. P. and Tiao, G. C. (1992). Bayesian Inference in Statistical Analysis. Wiley Classics Library. Wiley, New York.

Casella, G. and Berger, R. L. (2002). Statistical Inference, 2nd edition. Duxbury Advanced Series in Statistics and Decision Sciences. Thomson Learning, Pacific Grove, California.

Chib, S. and Greenberg, E. (1995). Understanding the metropolis-hastings algorithm. The American Statistician 49, 327-335.

Einstein, H. H. (2004). Decision aids for tunneling: update. Transportation Research Record: Journal of the Transportation Research Board, No. 1892, TRB, National Research Council, Washington, D.C., 2004, 199-207. 
Gelfand, A. E. and Smith, A. F. M. (1990). Sampling-based approaches to calculating marginal densities. Journal of the American Statistical Association 85, 398-409.

Lanney, N. A. (1978). Statistical Description of Rock Properties and Sampling. Thesis (M.S.) - Massachusetts Institute of Technology, Department of Civil and Environmental Engineering.

Lunn, D. J., Thomas, A., Best, N. and Spiegelhalter, D. (2000). Winbugs a Bayesian modelling framework: concepts, structure, and extensibility. Statistics and Computing 10, 325-337.

Merrien-Soukatchoff, V., Korini, T. and Thoraval, A. (2012). Use of an integrated discrete fracture network code for stochastic stability analyses of fractured rock masses. Rock Mechanics and Rock Engineering 45, 159-181.

Priest, S. D. (1985). Hemispherical Projection Methods in Rock Mechanics. Number of Pages 124. George Allen and Unwin, London.

Priest, S. D. (1993). Discontinuity Analysis for Rock Engineering. Chapman \& Hall/CRC, New York.

R Development Core Team (2012). R: A Language and Environment for Statistical Computing. R Foundation for Statistical Computing, Vienna, Austria. http://www.R-project.org.

Tierney, L. and Kadane, J. B. (1986). Accurate approximations for posterior moments and marginal densities. Journal of the American Statistical Association $\mathbf{8 1}, 82-86$.

Tierney, L., Kass, R. E. and Kadane, J. B. (1989). Fully exponential laplace approximations to expectations and variances of nonpositive functions. Journal of the American Statistical Association 84, 710-716.

Received February 24, 2013; accepted May 17, 2013.

Jorge Alberto Achcar

Departamento de Medicina Social

University of São Paulo

FMRP, Ribeirão Preto, SP, Brazil

jorge@icmc.usp.br 
Gian Franco Napa

Department of Geotechnical Engineering

University of São Paulo

EESC, São Carlos, SP, Brazil

gnapa@sc.usp.br

Roberto Molina De Souza

Coordenação da Matemática

Federal Technological University of Paraná

Cornélio Procópio, PR, Brazil

rmolinasouza@utfpr.edu.br 